\title{
Synthesis of Meso- and Racemic 1,3-Diamino-1,3-diphenylpropanes*)
}

\author{
Gwan Sun Lee, Thomas Kammermeier, Alexander Kaiser, Ernst Eibler, and Wolfgang Wiegrebe \\ Faculty of Chemistry and Pharmacy, Sonderforschungsbereich 234 der Deutschen Forschungsgemeinschaft, University, P.O. Box 397, D-8400 Regensburg
}

Received January 25, 1990

The Pt-complexes $\mathbf{2 a}, \mathbf{b}$ are synthesized according to Scheme 2 including oximation of and 1,4-addition of $\mathrm{H}_{2} \mathrm{~N}-\mathrm{OH}$ to the chalcone 3 and separation of the diastereomers either as bis-acetamides 5 or - less favourable - at the diamine stage 6.
Synthese von meso- und racemischen 1,3-Diamino-1,3-diphenylpropanen

Die Pt-Komplexe 2a, b wurden nach Schema 2 hergestellt, u.a. durch Oximierung und 1,4-Addition von $\mathrm{H}_{2} \mathrm{~N}-\mathrm{OH}$ an das Chalcon 3 und Trennung der Diastereomere entweder als Bis-Acetamide 5 oder - schwieriger - als Diamine 6.

Schönenberger et al. have reported on the Pt-complexes of 1,2-diamino1,2-diphenylethanes (Typ 1), cytostatic compounds with affinity to the estrogen receptor possessing a cisplatinum increment as a cytotoxic entity ${ }^{1)}$. Moreover, some of the ligands show estrogenic properties by themselves depending inter alia on their stereochemistry ${ }^{1)}$. Meso-1,2-Diamino-1,2-bis(2,6-dichloro-4-hydroxyphenyl)-ethane shows very remarkable tumor inhibiting effects in pharmacological tests ${ }^{1}$.

By correlation of ${ }^{1} \mathrm{H}$-NMR data defining the conformation of 1,2-diamino-1,2-diphenyl-Pt-complexes 1 with their ability to bind to the estrogen receptor Schönenberger et al. ${ }^{1}$ have found that the conception of an antiperiplanar arrangement of the aromatic increments in hexestrole (10 - 12 $\AA$ distance of the phenolic $\mathrm{OH}$-groups) cannot be transferred to estrogenic Pt-complexes (in erythro 1-(2,6-dichloro-4-hydroxyphenyl)-2-(2-chloro-4hydroxyphenyl)-1,2-diaminoethane-Pt-complex the phenyl groups are arranged synclinally).

Schönenberger et al. ${ }^{1)}$ discuss the contribution of $\lambda$ - and $\delta$-conformers of 5-membered Pt-complexes of type 1. The conformational flexibility of cyclic diamino-Pt-complexes should be enhanced when extending the 5-membered ring to a 6-membered one, in other words: comparing the efficacy of 1,3-diamino-1,3-diphenylpropane-Pt-complexes looks interesting. On the other hand the stability of the complexes<smiles>[R2]c1cc(C2NP(N)(Cl)(Cl)NC2C2=CCC([R1])C([R2])C2)ccc1P</smiles>

1 and the property of the (inorganic) leaving groups to be substituted by bionucleophiles should be of comparable magnitude in both classes of complexes.

Here we describe the synthesis of the diastereomeric complexes $2 \mathbf{a}, \mathbf{b}$ which are to be compared with the analogously substituted 1,2-diamino-1,2-diphenylethanes $1 \mathbf{1 a}, \mathbf{b}^{2)}$ as far as their biochemical and pharmacological properties are concerned.

The strategies of Schönenberger's group for the synthesis of compounds $1^{3)}$ (aza-Cope-rearrangements of stilbenes) cannot be used for the preparation of compounds 2 . The chalcones 3 were obtained by $\mathrm{OH}^{-}$-catalyzed condensation of anisaldehyde with p-methoxyacetophenone (for 3a) following the general procedure of Kohler $^{4)}$. - 3,4-Dimethoxybenzaldehyde and 3,4-dimethoxyacetophenone were used for $3 \mathrm{~b}$. Reaction of compounds 3 with 2 moles of $\mathrm{H}_{2} \mathrm{~N}-\mathrm{OH}$ under basic conditions led to the hydroxyamino-hydroxyiminocompounds 4 (method: Arakawa ${ }^{5}$, v. Auwers ${ }^{6}$ ), which were reduced with $\mathrm{NaBH}_{4} / \mathrm{TiCl}_{4}{ }^{7)}$ to afford the bis-amides 5 after acetylation. At this stage of the reaction sequence the diastereomers of 5 (meso/racemic) were separated.

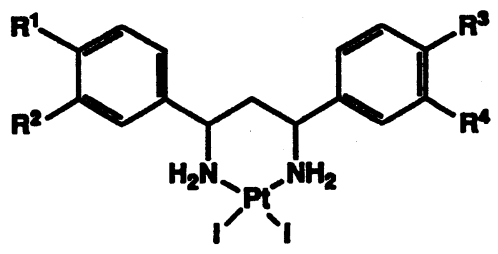

2
12: $R^{1}=R^{3}=O H, R^{2}=R^{4}=H$

1b: $R^{1}=R^{2}=R^{3}=R^{4}=O H$ 2a: $R^{1}=R^{3}=O H, R^{2}=R^{4}=H$

20. $: R^{1} \cdot R^{4}=O H$

\footnotetext{
${ }^{*}$ Dedicated to Prof. Dr. H. Möhrle, Düsseldorf, on the occasion of his 60th birthday.
} 


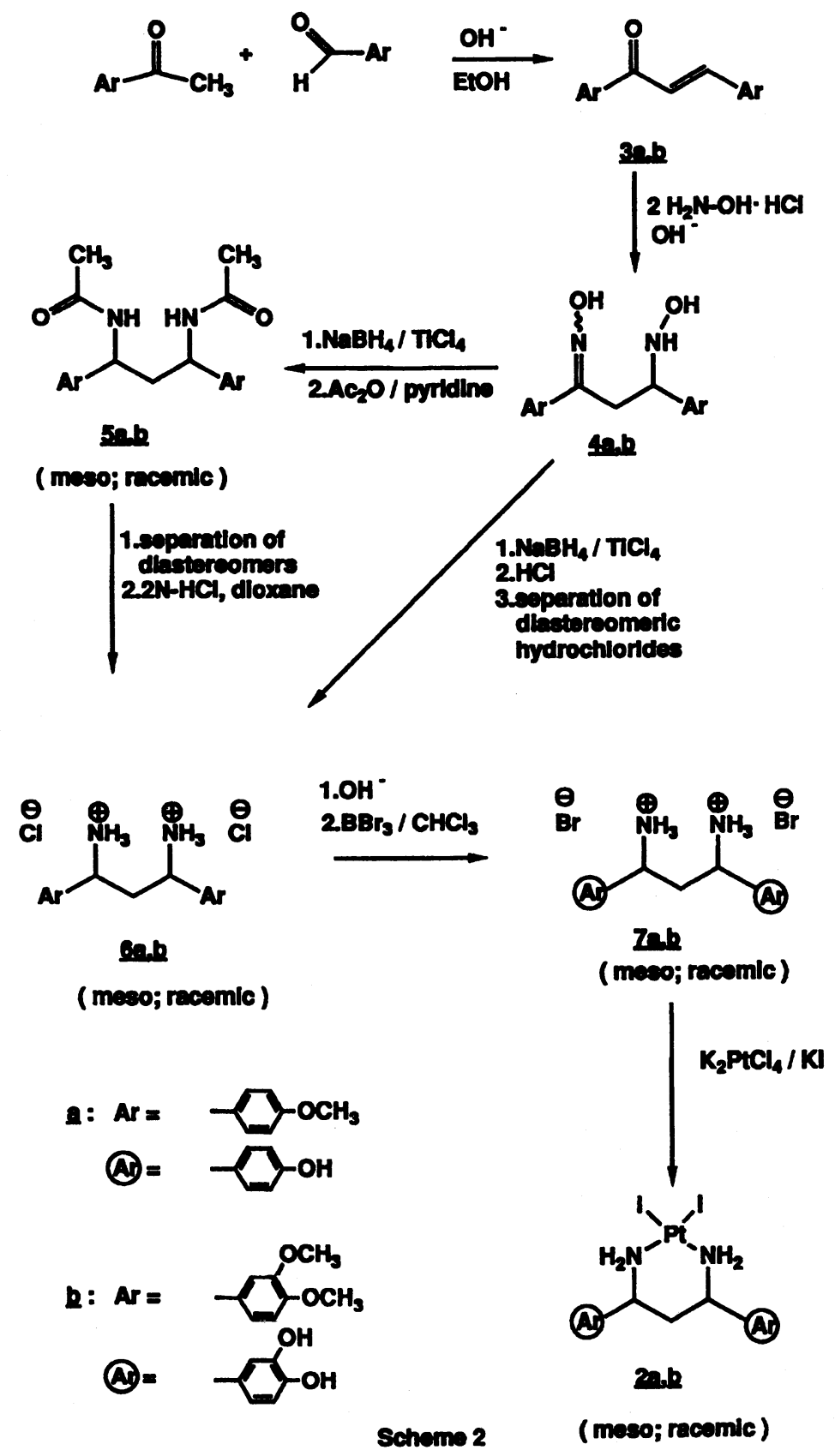

In the course of the conversion of compounds 4 to the bis-acetamides 5 the crude diastereomeric bis-amines 6 are acetylated for separation. - Alternatively the isomers of $6 a$ can be separated by fractional crystallization as di-hydrochlorides from EtOH (meso-6a.2 $\mathrm{HCl}$ ) and glacial acetic acid (racem.-6a.2 $\mathrm{HCl}$ ), respectively. This procedure does not work with $\mathbf{6 b}$.

In the case of $5 \mathrm{a}$ the diastereomers were assigned meso or racemic, respectively, by reason of their ${ }^{1} \mathrm{H}-\mathrm{NMR}$-spectra showing the multiplet for the $\mathrm{CH}_{2}$-group of meso-5, and the triplet of racem.-5, contrary to lit. ${ }^{5)}$. - Unequivocal assignment stems from separation of one diastereomer into enantiomers (racem.-5) by HPLC on a chiral column (Fig. 1) (cf. Experimental Part), whilst the other diastereomer remained unresolved under identical conditions (meso-5). - The hydroxyamino-hydroxyimino-compound $4 \mathrm{a}$ was reduced and separated to the diastereomers of 6 without acetylation. After converting the bis-hydrochlorides of compounds 6 to the free bases (a critical step!), the bisamines 6 were cleaved to the biphenols 7 by $\mathrm{BBr}_{3}$, yielding compounds 7 as bis-hydrobromides. Treatment with $\mathrm{K}_{2} \mathrm{PtCl}_{4} / \mathrm{KI}$ afforded the Pt-complexes 2a, b. Curiously enough this step works nicely with the racemic diastereomers whilst meso-7a, $\mathbf{b}$ are obtained after laborious work-up only.

During the preparation of $\mathbf{4 b}$ the corresponding bis-hydroxyimino-analogue (formal dehydrogenation product of 4b with two oxime increments) was obtained as a by-product $\left(\mathrm{cf}{ }^{5) 6}\right)$. This mixture, however, when being reduced 


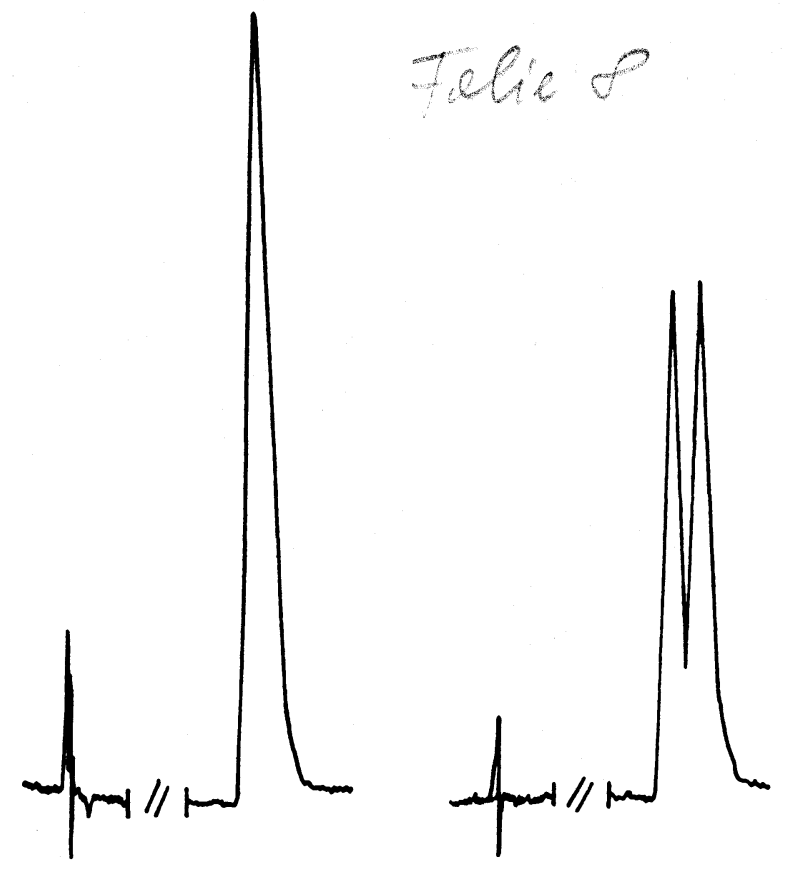

Fig. 1: HPLC-experiments with meso-5a (left) and racem.-5a (right) on a chiral column.

with $\mathrm{NaBH}_{4} / \mathrm{TiCl}_{4}$, followed by acetylation, afforded $5 \mathbf{b}$ as a mixture of diastereomers, which was separated as described for 5 a.

\section{Experimental Part}

General procedures: m. ps. (uncorrected): apparatus according to Dr. Tottoli (Büchi). - Elemental analysis: Microanalysis Laboratory, University of Regensburg. - IR-spectra (in $\mathrm{KBr}$ ): Beckman Acculab 3. - ${ }^{1} \mathrm{H}-\mathrm{NMR}$ spectra: Varian EM $390(90 \mathrm{MHz})$ or Bruker WM 250 (250 MHz). - Mass spectra: Varian MAT CH 5.

\section{1,3-Bis-(4'-methoxyphenyl)-2-propen-1-one (3a)}

To a stirred mixture of $\mathrm{NaOH}(5.07 \mathrm{~g}, 0.1265 \mathrm{~mole})$ in $\mathrm{H}_{2} \mathrm{O}(45 \mathrm{ml})$ and $\mathrm{EtOH}(29 \mathrm{ml})$ at $15^{\circ} \mathrm{C}$ were added 4-methoxyacetophenone $(15.02 \mathrm{~g}, 0.1$ mole) and 4-methoxybenzaldehyde (13.62 g, 0.1 mole). The mixture was stirred for $12 \mathrm{~h}$ at room temp. and filtered. The filter cake was washed with $\mathrm{EtOH} / \mathrm{H}_{2} \mathrm{O}(1: 1, \mathrm{v} / \mathrm{v} ; 20 \mathrm{ml})$ and dried to afford 3a as a light yellow solid. Yield $25.5 \mathrm{~g}(95 \%)$ - m.p. $101-102^{\circ} \mathrm{C}$, Lit. $\left.{ }^{8}\right)$ : m.p. 101-102 ${ }^{\circ} \mathrm{C}$. - IR (KBr, $\left.\mathrm{cm}^{-1}\right): 1600(\mathrm{C}=\mathrm{C}) ; 1665(\mathrm{C}=0)$. $-{ }^{1} \mathrm{H}-\mathrm{NMR}\left(\mathrm{CDCl}_{3}\right): \delta(\mathrm{ppm})=3.80(\mathrm{~s}$; $\left.3 \mathrm{H},-\mathrm{OCH}_{3}\right), 3.84\left(\mathrm{~s} ; 3 \mathrm{H},-\mathrm{OCH}_{3}\right), 6.83-8.13(\mathrm{~m} ; 10 \mathrm{H}, 8 \mathrm{Ar}-\mathrm{H}$ and 2 olefinic $\mathrm{H})$.

\section{1,3-Bis-(3',4'-dimethoxyphenyl)-2-propen-1-one (3b)}

3b was prepared as described for 3a. Yield 94\%. - m.p. 116-118 ${ }^{\circ} \mathrm{C}$, Lit. ${ }^{9)}$ : m.p. $116-118^{\circ} \mathrm{C}$. - IR (KBr, cm $\left.{ }^{-1}\right): 1605(\mathrm{C}=\mathrm{C}) ; 1660(\mathrm{C}=0)$. - ${ }^{1} \mathrm{H}-$ $\mathrm{NMR}\left(\mathrm{CDCl}_{3}\right): \delta(\mathrm{ppm})=3.91,3.94\left(2 \mathrm{~s} ; 12 \mathrm{H},-\mathrm{OCH}_{3}\right), 6.87-7.88(\mathrm{~m} ; 8 \mathrm{H}$, $6 \mathrm{Ar}-\mathrm{H}$ and 2 olefinic $\mathrm{H})$.

\section{1,3-Bis-(4'-methoxyphenyl)-3-hydroxyamino-1-hydroxyiminopropane (4a)}

To a stirred solution of $3 \mathrm{a}(26.83 \mathrm{~g}, 0.1 \mathrm{~mole})$ in $\mathrm{EtOH}(240 \mathrm{ml})$ at $50^{\circ} \mathrm{C}$ were added $\mathrm{NH}_{2} \mathrm{OH} \cdot \mathrm{HCl}(18.3 \mathrm{~g}, 0.263 \mathrm{~mole})$ in $\mathrm{H}_{2} \mathrm{O}(40 \mathrm{ml})$ and $\mathrm{KOH}$ ( $24 \mathrm{~g}, 0.42 \mathrm{~mole})$ in $\mathrm{H}_{2} \mathrm{O}(40 \mathrm{ml})$. The resulting solution was refluxed for
$20 \mathrm{~min}$ and cooled to room temp. $\mathrm{H}_{2} \mathrm{O}$ (1.5 l) was added and the mixture was stirred at $0-5^{\circ} \mathrm{C}$ for $1 \mathrm{~h}$. The solid was filtered, stirred in $\mathrm{CH}_{2} \mathrm{Cl}_{2}(100$ $\mathrm{ml})$ for $20 \mathrm{~min}$ and filtered again. Crystallization from $\mathrm{MeOH}(220 \mathrm{ml})$ afforded $4 \mathrm{a}$ as white crystals. Yield $14.0 \mathrm{~g}(44 \%)$. - m.p. 133-134 ${ }^{\circ} \mathrm{C}$. - IR $\left(\mathrm{KBr}, \mathrm{cm}^{-1}\right)$ : $1610(\mathrm{C}=\mathrm{N}) ; 2800-3500$ (broad, $\mathrm{NH}$ and $\left.\mathrm{OH}\right) .{ }^{-} \mathrm{H}-\mathrm{NMR}$ $\left(\mathrm{CDCl}_{3}+\mathrm{DMSO}_{-} \mathrm{d}_{6}\right): \delta(\mathrm{ppm})=2.83-3.50\left(\mathrm{~m}: 2 \mathrm{H},-\mathrm{CH}_{2}-\right), 3.73(\mathrm{~s} ; 3 \mathrm{H}$, $\left.-\mathrm{OCH}_{3}\right), 3.77$ (s; $\left.3 \mathrm{H},-\mathrm{OCH}_{3}\right), 4.20$ (t; J = 7.5 Hz, $\left.1 \mathrm{H},-\mathrm{CH}-\right), 5.33$ (broad s; $1 \mathrm{H}, \mathrm{D}_{2} \mathrm{O}$-exchange), 6.67-7.60 (m; $\left.8 \mathrm{H}, \mathrm{Ar}-\mathrm{H}\right), 7.20$ (broad s; $1 \mathrm{H}, \mathrm{D}_{2} \mathrm{O}-$ exchange), 10.77 (broad s; $1 \mathrm{H}, \mathrm{D}_{2} \mathrm{O}$-exchange). - El-MS: $\mathrm{m} / \mathrm{z}=316(7 \%$, $\left.\mathrm{M}^{+\cdot}\right), 298\left(7,\left(\mathrm{M}-\mathrm{H}_{2} \mathrm{O}\right)^{+\cdot}\right), 165\left(23,\left(\mathrm{M}-{ }^{\circ} \mathrm{C}_{8} \mathrm{H}_{9} \mathrm{NO}_{2}\right)^{+}\right.$, McLafferty), 152 (100, $\left(\mathrm{M}-{ }^{\circ} \mathrm{C}_{9} \mathrm{H}_{10} \mathrm{NO}_{2}\right)^{+}$, benzyl- and $\beta$-cleavage). $-\mathrm{C}_{17} \mathrm{H}_{20} \mathrm{~N}_{2} \mathrm{O}_{4}$ (316.4) Calc. C 64.5 H 6.37 N 8.9 Found C 64.4 H 6.40 N 8.8.

1,3-Bis-(3',4'-dimethoxyphenyl)-3-hydroxyamino-1-hydroxyiminopropane (4b) and 1,3-Bis-(3',4'-dimethoxyphenyl)-propane-1,3-dioxime

$4 \mathrm{~b}$ was prepared analogously to $4 \mathrm{a}$; but a mixture of $4 \mathrm{~b}$ and the formal dehydrogenation product (bisoxime, no number) was obtained. Crystallization from EtOH (99\%) afforded the pure dioxime, whilst crystallization from toluene led to pure $\mathbf{4 b}$. In these crystallization procedures the respective other component of the mixture remained in the mother liquors in each case. Yield $21.2 \mathrm{~g}$ of the mixture.

4b: m.p. $175-178^{\circ} \mathrm{C}$. - IR $\left(\mathrm{KBr}, \mathrm{cm}^{-1}\right): 1610(\mathrm{C}=\mathrm{N}), 3600-3100$ (broad, $\mathrm{NH}$ and $\mathrm{OH})$. - ${ }^{1} \mathrm{H}-\mathrm{NMR}\left(\mathrm{CDCl}_{3}+\mathrm{DMSO}_{-} \mathrm{d}_{6}\right): \delta(\mathrm{ppm})=2.93-3.27(\mathrm{~m}$; $\left.2 \mathrm{H},-\mathrm{CH}_{2}-\right), 3.70,3.75\left(2 \mathrm{~s} ; 12 \mathrm{H},-\mathrm{OCH}_{3}\right), 4.15(\mathrm{t} ; \mathrm{J}=7.5 \mathrm{~Hz}, 1 \mathrm{H},-\mathrm{CH}-)$, 5.55 (broad s; 1H, $\mathrm{D}_{2} \mathrm{O}$-exchange), 6.75-7.02 (m; 6H, Ar-H), $7.18(\mathrm{~s} ; 1 \mathrm{H}$, $\mathrm{D}_{2} \mathrm{O}$-exchange), 10.89 (s; $1 \mathrm{H}, \mathrm{D}_{2} \mathrm{O}$-exchange). - EI-MS: $\mathrm{m} / \mathrm{z}=376(10 \%$, $\left.\mathrm{M}^{+{ }^{+}}\right), 358\left(7,\left(\mathrm{M}-\mathrm{H}_{2} \mathrm{O}\right)^{+}\right), 195\left(100,\left(\mathrm{M}-{ }^{\circ} \mathrm{C}_{9} \mathrm{H}_{11} \mathrm{NO}_{3}\right)^{+}\right.$, McLafferty), 181 (83, $\left(\mathrm{M}-{ }^{\circ} \mathrm{C}_{10} \mathrm{H}_{13} \mathrm{NO}_{3}\right)^{+}$, McLafferty). - $\mathrm{C}_{19} \mathrm{H}_{24} \mathrm{~N}_{2} \mathrm{O}_{6}$ (376.4) Calc. C 60.6 H 6.43 N 7.4 Found C 60.6 H 6.21 N 7.1.

\section{1,3-Bis-(3',4'-dimethoxyphenyl)-propane-1,3-dioxime $\cdot 0.5 \mathrm{C}_{2} \mathrm{H}_{5} \mathrm{OH}$}

M.p. $187-189^{\circ} \mathrm{C}$. - IR (KBr, cm $\left.{ }^{-1}\right): 1610(\mathrm{C}=\mathrm{N}) ; 3400(\mathrm{OH}) .-^{1} \mathrm{H}-\mathrm{NMR}$ $\left(\mathrm{CDCl}_{3}+\mathrm{DMSO}_{-} \mathrm{d}_{6}\right): \delta(\mathrm{ppm})=1.10\left(\mathrm{t} ; \mathrm{J}=7.5 \mathrm{~Hz}, 1.5 \mathrm{H}, 0.5 \mathrm{CH}_{3}-\right), 2.50$ (s; 0.5H, 0.5-OH, $\mathrm{D}_{2} \mathrm{O}$-exchange), 3.50 (q; $\mathrm{J}=7 \mathrm{~Hz}, 1 \mathrm{H}, 0.5-\mathrm{CH}_{2}-$ ), 3.72, $3.74\left(2 \mathrm{~s} ; 12 \mathrm{H},-\mathrm{OCH}_{3}\right), 4.32\left(\mathrm{~s} ; 2 \mathrm{H},-\mathrm{CH}_{2}-\right), 6.75-7.20(\mathrm{~m} ; 6 \mathrm{H}, \mathrm{Ar}-\mathrm{H})$, 11.33 (s; 2H, -OH, $\mathrm{D}_{2} \mathrm{O}$-exchange). - NI-FAB-MS (glycerol/DMSO): $\mathrm{m} / \mathrm{z}$ $=374$ (23\%), $373\left(100,(\mathrm{M}-\mathrm{H})^{-}\right) .-\mathrm{C}_{19} \mathrm{H}_{22} \mathrm{O}_{6} \mathrm{~N}_{2} \cdot 0.5 \mathrm{C}_{2} \mathrm{H}_{5} \mathrm{OH}$ (397.4) Calc. C 60.4 H 6.34 N 7.0 Found C 60.1 H 6.24 N 6.7.

\section{1,3-Bis-(4'-methoxyphenyl)-1,3-diacetaminopropane (5a)}

To a stirred solution of $\mathrm{TiCl}_{4}(33.7 \mathrm{ml}, 0.307$ mole $)$ in dry 1,2-dimethoxyethane $(370 \mathrm{ml})$ was added $\mathrm{NaBH}_{4}(23.4 \mathrm{~g}, 0.613$ mole) cautiously at $0^{\circ} \mathrm{C}$ under $\mathrm{N}_{2}$. The resulting dark blue solution was stirred for $30 \mathrm{~min}$ at $0^{\circ} \mathrm{C}$ and $4 \mathrm{a}(23.15 \mathrm{~g}, 0.073 \mathrm{~mole})$ in dry 1,2-dimethoxyethane $(350 \mathrm{ml})$ was added dropwise for $30 \mathrm{~min}$. The mixture was stirred at room temp. for $20 \mathrm{~h}$ and cooled to $0^{\circ} \mathrm{C} . \mathrm{H}_{2} \mathrm{O}(300 \mathrm{ml})$ was added drop by drop and the resulting solution was neutralized with conc. aqueous $\mathrm{NH}_{3}(180 \mathrm{ml})$. To the suspension were added EtOAc (11) and $\mathrm{H}_{2} \mathrm{O}$ (11), and stirring was continued for $1 \mathrm{~h}$. The mixture was filtered through celite and the upper layer was separated. The aqueous layer was further extracted with EtOAc $(2 \times 250 \mathrm{ml})$. The combined org. layers were washed with saturated $\mathrm{NaCl}$ solution, dried over $\mathrm{Na}_{2} \mathrm{SO}_{4}$, filtered, and evaporated in vacuo.

The residue was dissolved in $\mathrm{CH}_{2} \mathrm{Cl}_{2}(1.4 \mathrm{l})$ and cooled to $0^{\circ} \mathrm{C}$. Pyridine (17.05 ml, 0.219 mole) and $\mathrm{Ac}_{2} \mathrm{O}(20.44 \mathrm{ml}, 0.219$ mole) were added, the mixture was stirred for $2 \mathrm{~h}$ at room temp., washed with $\mathrm{N}-\mathrm{HCl}\left(\begin{array}{ll}1 & 1\end{array}\right), \mathrm{H}_{2} \mathrm{O}$ (1 1) and saturated $\mathrm{NaCl}$ solution (1 l) and dried over $\mathrm{Na}_{2} \mathrm{SO}_{4}$. After evaporating the solvent, the two diastereomeres were separated by $\mathrm{CC}$ $\left(\mathrm{CHCl}_{3} / \mathrm{MeOH}(10 / 1 ; \mathrm{v} / \mathrm{v})\right)$ and identified by HPLC on a chiral column (see below); the first crop was found to be the meso-isomer, the second one to be the racem.-isomer. 


\section{HPLC-resolution of racem.-5a}

Conditions: Column: Bakerbond Covalent Chiral, 5 (250 mm x $4.6 \mathrm{~mm}$ ). - Eluent: hexane/2-propanol/MeOH = 85/10/5 (v/v/v; plus 4 drops of conc. $\mathrm{NH}_{3} / 100 \mathrm{ml}$ of eluent). - Flow rate: $0.8 \mathrm{ml} / \mathrm{min}$. - Detector: UV $(254 \mathrm{~nm})$.

meso-5a: Yield $4.36 \mathrm{~g}(16 \%)$ - m.p. 250-251 ${ }^{\circ} \mathrm{C}(\mathrm{EtOH} 99 \%)$. - $\mathrm{IR}(\mathrm{KBr}$, $\left.\mathrm{cm}^{-1}\right)$ : $1660(\mathrm{C}=0) ; 3310(\mathrm{NH}) .-{ }^{1} \mathrm{H}-\mathrm{NMR}\left(\mathrm{CDCl}_{3}+\mathrm{DMSO}_{6}\right): \delta(\mathrm{ppm})$ $=1.81\left(\mathrm{~s} ; 6 \mathrm{H},-\mathrm{COCH}_{3}\right), 1.87-2.12\left(\mathrm{~m} ; 2 \mathrm{H},-\mathrm{CH}_{2}-\right), 3.72\left(\mathrm{~s} ; 6 \mathrm{H},-\mathrm{OCH}_{3}\right)$, 4.48-4.57 (m; 2H, -CH-), 6.96 (m; AA 'BB', $8 \mathrm{H}, \mathrm{Ar}-\mathrm{H}$ ), 8.30 (d; J $=8.4 \mathrm{~Hz}$, 2H, -NH-), - EI-MS: $\mathrm{m} / \mathrm{z}=370\left(6 \%, \mathrm{M}^{+*}\right), 311\left(11,\left(\mathrm{M}-\mathrm{CH}_{3} \mathrm{CONH}_{2}\right)^{+}\right.$, McLafferty), 268 (15, (311 - $\left.\mathrm{CH}_{3} \mathrm{CO}\right), 192$ (74, (M - Ar-CH-NHAc) $\left.{ }^{+}\right), 178$ (36, $\left.(\mathrm{Ar}-\mathrm{CH}=\mathrm{NH}-\mathrm{Ac})^{+}\right), 150\left(42,\left(192-\mathrm{CH}_{2}=\mathrm{C}=0\right), 136\right.$ (100, (Ar$\left.\left.\mathrm{CH}=\mathrm{NH}_{2}\right)^{+}\right) .-\mathrm{C}_{21} \mathrm{H}_{26} \mathrm{~N}_{2} \mathrm{O}_{4}(370.4)$ Calc. C 68.1 H 7.07 N 7.6 Found $\mathrm{C}$ 67.7 H 7.41 N 7.2.

racem.-5a: Yield $7.60 \mathrm{~g}(28 \%)$ - m.p. $228-229^{\circ} \mathrm{C}$ (EtOH 99\%). - IR $\left(\mathrm{KBr}, \mathrm{cm}^{-1}\right): 1645(\mathrm{C}=0)$ ); $3260(\mathrm{NH}) .{ }^{1} \mathrm{H}-\mathrm{NMR}$ (DMSO-d $\left.\mathrm{d}_{6}\right): \delta(\mathrm{ppm})=$ $1.70\left(\mathrm{~s} ; 6 \mathrm{H},-\mathrm{COCH}_{3}\right), 1.96\left(\mathrm{t} ; \mathrm{J}=7.7 \mathrm{~Hz}, 2 \mathrm{H},-\mathrm{CH}_{2}-\right), 3.72(\mathrm{~s} ; 6 \mathrm{H}$, $\left.\mathrm{OCH}_{3}\right), 4.67-4.76(\mathrm{~m} ; 2 \mathrm{H},-\mathrm{CH}-), 7.01\left(\mathrm{~m} ; \mathrm{AA}^{\prime} \mathrm{BB}^{\prime}, 8 \mathrm{H}, \mathrm{Ar}-\mathrm{H}\right), 8.22$ (d; $\mathrm{J}=8.3 \mathrm{~Hz}, 2 \mathrm{H},-\mathrm{NH}-)$. - EI-MS: $\mathrm{m} / \mathrm{z}=370\left(16, \mathrm{M}^{+{ }^{+}}\right), 311$ (26, (M $\left.\mathrm{CH}_{3} \mathrm{CONH}_{2}\right)^{+}$, McLafferty), 268 (33, (311 - $\left.\mathrm{CH}_{3} \mathrm{CO}\right), 192$ (94, (M - ArCH-NHAc) $\left.)^{+}\right), 178\left(47,(\mathrm{Ar}-\mathrm{CH}=\mathrm{NH}-\mathrm{Ac})^{+}\right), 150\left(51,\left(192-\mathrm{CH}_{2}=\mathrm{C}=0\right)\right.$, $136\left(100,\left(\mathrm{Ar}-\mathrm{CH}=\mathrm{NH}_{2}\right)^{+}\right)$. $-\mathrm{C}_{21} \mathrm{H}_{26} \mathrm{~N}_{2} \mathrm{O}_{4}(370.4)$ Calc. C $68.1 \mathrm{H} 7.07 \mathrm{~N}$ 7.6 Found $\mathrm{C} 67.8 \mathrm{H} 7.17 \mathrm{~N}$ 7.6.

\section{1,3-Bis-(3',4'-dimethoxyphenyl)-1,3-diacetaminopropane (5b)}

$\mathbf{5 b}$ was prepared in the same way as $\mathbf{5 a}$, but the mixture was extracted with $\mathrm{CH}_{2} \mathrm{Cl}_{2}$ instead of EtOAc and the eluent for $\mathrm{CC}$ was acetone/ $\mathrm{CH}_{2} \mathrm{Cl}_{2}$ $(1 / 1 ; v / v)$.

meso-5b: Yield 6\%. - m.p. 187-188 ${ }^{\circ} \mathrm{C}$ (toluene/EtOAc (1/4; v/v)). - IR $\left(\mathrm{KBr}, \mathrm{cm}^{-1}\right): 1650(\mathrm{C}=0) ; 3260(\mathrm{NH}) .{ }^{1} \mathrm{H}-\mathrm{NMR}\left(\mathrm{CDCl}_{3}\right): \delta(\mathrm{ppm})=1.99$ $\left(\mathrm{s} ; 6 \mathrm{H},-\mathrm{COCH}_{3}\right), 2.12-2.47\left(\mathrm{~m} ; 2 \mathrm{H},-\mathrm{CH}_{2}-\right), 3.84,3.85\left(2 \mathrm{~s} ; 12 \mathrm{H},-\mathrm{OCH}_{3}\right)$, 4.78-4.86 (m; 2H, -CH-), 6.32 (d; J = 7.4 Hz, 2H, -NH-), 6.77-6.81 (m; 6H, Ar-H). - EI-MS: $\mathrm{m} / \mathrm{z}=430\left(13, \mathrm{M}^{+}\right), 387\left(4,\left(\mathrm{M}-\mathrm{COCH}_{3}\right)^{+}\right), 371(14,(\mathrm{M}$ - $\left.\mathrm{CH}_{3} \mathrm{CONH}_{2}\right)^{+}$, McLafferty), 328 (27, (371 - $\left.\mathrm{CH}_{3} \mathrm{CO}\right), 222$ (100, (M $\left.\mathrm{Ar}-\mathrm{CH}-\mathrm{NH}-\mathrm{Ac})^{+}\right), 208 \quad\left(28, \quad(\mathrm{Ar}-\mathrm{CH}=\mathrm{NH}-\mathrm{Ac})^{+}\right), \quad 180 \quad(57, \quad(222$ $\mathrm{CH}_{2}=\mathrm{C}=0$ ), $166\left(86,\left(\mathrm{Ar}-\mathrm{CH}=\mathrm{NH}_{2}\right)^{+}\right) .-\mathrm{C}_{23} \mathrm{H}_{30} \mathrm{~N}_{2} \mathrm{O}_{6}(430.5)$ Calc. $\mathrm{C} 64.2$ H 7.02 N 6.5 Found C 63.8 H 6.82 N 6.4.

racem.-5b: Yield 10\%. - m.p. 189-190 ${ }^{\circ} \mathrm{C}$ (toluene/EtOAc (1/4; v/v)). - IR $\left(\mathrm{KBr}, \mathrm{cm}^{-1}\right): 1645(\mathrm{C}=0) ; 3260(\mathrm{NH}) .{ }^{1} \mathrm{H}-\mathrm{NMR}\left(\mathrm{CDCl}_{3}\right): \delta(\mathrm{ppm})=1.93$ (s; $\left.6 \mathrm{H},-\mathrm{COCH}_{3}\right), 2.47$ (t; J $\left.=7.2 \mathrm{~Hz}, 2 \mathrm{H},-\mathrm{CH}_{2}-\right), 3.83,3.85(2 \mathrm{~s} ; 12 \mathrm{H}$, $\left.-\mathrm{OCH}_{3}\right), 4.67-4.76(\mathrm{~m} ; 2 \mathrm{H},-\mathrm{CH}-), 5.97(\mathrm{~d} ; \mathrm{J}=7.5 \mathrm{~Hz}, 2 \mathrm{H},-\mathrm{NH}-), 6.73-$ $6.82(\mathrm{~m} ; 6 \mathrm{H}, \mathrm{Ar}-\mathrm{H})$. - EI-MS: $\mathrm{m} / \mathrm{z}=430\left(11, \mathrm{M}^{+{ }^{+}}\right), 387(6,(\mathrm{M}$ $\left.\left.\mathrm{COCH}_{3}\right)^{+}\right) ; 371\left(16,\left(\mathrm{M}-\mathrm{CH}_{3} \mathrm{CONH}_{2}\right)^{+}, \mathrm{McLafferty}\right), 328$ (27, (371 $\left.\mathrm{CH}_{3} \mathrm{CO}\right), 222\left(100,(\mathrm{M}-\mathrm{Ar}-\mathrm{CH}-\mathrm{NH}-\mathrm{Ac})^{+}\right), 208\left(25,(\mathrm{Ar}-\mathrm{CH}=\mathrm{NH}-\mathrm{Ac})^{+}\right)$, $180\left(65,\left(222-\mathrm{CH}_{2}=\mathrm{C}=0\right), 166\left(92,\left(\mathrm{Ar}-\mathrm{CH}=\mathrm{NH}_{2}\right)^{+}\right) .-\mathrm{C}_{23} \mathrm{H}_{30} \mathrm{~N}_{2} \mathrm{O}_{6}\right.$ (430.5) Calc. C 64.2 H 7.02 N 6.5 Found C 63.9 H 6.83 N 6.3

\section{1,3-Bis-(4'-methoxyphenyl)-1,3-diaminopropane dihydrochloride (6a)}

meso-6a: The stirred mixture of meso-5a $(1.2 \mathrm{~g}, 3.24 \mathrm{mmole})$ in $2 \mathrm{~N}-\mathrm{HCl}$ $(60 \mathrm{ml})$ and dioxane $(30 \mathrm{ml})$ was refluxed for $12 \mathrm{~h}$ and cooled to room temp. The solvents were evaporated in vacuo and the residue was stirred in acetone $(30 \mathrm{ml})$, filtered and crystallized from $\mathrm{AcOH} / \mathrm{H}_{2} \mathrm{O}(10 / 1 ; \mathrm{v} / \mathrm{v})$. The crystals were filtered, washed with acetone $(10 \mathrm{ml})$, and dried to afford meso-6a.2 $\mathrm{HCl}$ as white crystals. Yield $0.73 \mathrm{~g}(63 \%)$ - m.p. $277-282^{\circ} \mathrm{C}$ (dec.). - IR (KBr, cm ${ }^{-1}$ ): 1620 (aromatic $\mathrm{C}=\mathrm{C}$ ); $2600-3400(\mathrm{NH}),-{ }^{1} \mathrm{H}$ $\operatorname{NMR}\left(\mathrm{D}_{2} \mathrm{O}\right): \delta(\mathrm{ppm})=2.60-2.93\left(\mathrm{~m} ; 2 \mathrm{H},-\mathrm{CH}_{2}-\right), 3.70\left(\mathrm{~s} ; 6 \mathrm{H},-\mathrm{OCH}_{3}\right)$, 4.27-4.50 (m; 2H, -CH-), 6.83 (m; AA'BB', 8H, Ar-H). $\mathrm{C}_{17} \mathrm{H}_{22} \mathrm{~N}_{2} \mathrm{O}_{2} \cdot 2 \mathrm{HCl}(359.3)$ Calc. C $56.8 \mathrm{H} 6.73 \mathrm{~N} 7.8$ Found C $56.6 \mathrm{H} 6.74$ N 7.7.

racem.-6a: Same reaction as described for meso-6a using racem.-5a $(2.3 \mathrm{~g}, 6.2 \mathrm{mmole})$ in $2 \mathrm{~N}-\mathrm{HCl}(110 \mathrm{ml})$ and dioxane $(55 \mathrm{ml})$. Yield $1.35 \mathrm{~g}$
(61\%). - m.p. $255-260^{\circ} \mathrm{C}$ (dec., glacial AcOH). - IR (KBr, cm $\left.{ }^{-1}\right): 1620$ (aromatic $\mathrm{C}=\mathrm{C})$; $2600-3400(\mathrm{NH})$. - ${ }^{1} \mathrm{H}-\mathrm{NMR}\left(\mathrm{D}_{2} \mathrm{O}\right)$ : $\delta(\mathrm{ppm})=\mathbf{2 . 6 0 - 2 . 8 0}$ (m; $2 \mathrm{H},-\mathrm{CH}_{2}-$ ), 3.70-3.90 (m; $\left.2 \mathrm{H},-\mathrm{CH}-\right), 3.75\left(\mathrm{~s} ; 6 \mathrm{H},-\mathrm{OCH}_{3}\right), 7.10$ (m; AA'BB', 8H, Ar-H). - $\mathrm{C}_{17} \mathrm{H}_{22} \mathrm{~N}_{2} \mathrm{O}_{2} \cdot 2 \mathrm{HCl}(359.3)$ Calc. C $56.8 \mathrm{H} 6.73 \mathrm{~N}$ 7.8 Found $\mathrm{C} 56.6 \mathrm{H} 6.88 \mathrm{~N} 7.5$.

\section{1,3-Bis-(3',4'-dimethoxyphenyl)-1,3-diaminopropane dipicrate (6b)}

The dihydrochlorides of $6 \mathrm{~b}$ were prepared analogously to those of $6 \mathrm{a}$. As it was not possible to purify them, the dipicrates were analyzed.

meso-6b-dipicrate: Yield 58\%. - m.p. 193-198 ${ }^{\circ} \mathrm{C}$ (dec., glacial AcOH/ $\left./ \mathrm{H}_{2} \mathrm{O}(1 / 10 ; \mathrm{v} / \mathrm{v})\right)$. - IR (KBr, cm $\left.{ }^{-1}\right): 2800-3600$ (broad, NH). - ${ }^{1} \mathrm{H}$ NMR (DMSO-d $): \delta(\mathrm{ppm})=2.51$ (broad s; $\left.2 \mathrm{H},-\mathrm{CH}_{2}-\right), 3.65(\mathrm{~s} ; 6 \mathrm{H}$, $\left.\mathrm{OCH}_{3}\right), 3.72\left(\mathrm{~s} ; 6 \mathrm{H},-\mathrm{OCH}_{3}\right), 4.13$ (broad s: $\left.2 \mathrm{H},-\mathrm{CH}-\right), 6.78-6.88$ (m; $6 \mathrm{H}$, Ar-H), 8.24 (braod s; $6 \mathrm{H},-\mathrm{NH}_{3}{ }^{+}, \mathrm{D}_{2} \mathrm{O}$-exchange), 8.60 (s; $\left.4 \mathrm{H}, \mathrm{Ar}-\mathrm{H}\right)$. $\mathrm{C}_{19} \mathrm{H}_{26} \mathrm{~N}_{2} \mathrm{O}_{4} \cdot 2 \mathrm{C}_{6} \mathrm{H}_{3} \mathrm{~N}_{3} \mathrm{O}_{7} \cdot \mathrm{H}_{2} \mathrm{O}(822.7)$ Calc. C $45.3 \mathrm{H} 4.17 \mathrm{~N} 13.6$ Found $\mathrm{C}$ 45.4 H 4.24 N 13.2.

racem.-6b-dipicrate: Yield 63\%. - m.p. 212-217 ${ }^{\circ} \mathrm{C}$ (dec., glacial $\mathrm{AcOH} / \mathrm{H}_{2} \mathrm{O}$ 1/10 (v/v)). - IR ( $\mathrm{KBr}, \mathrm{cm}^{-1}$ ): 2800-3600 (broad, NH). - ${ }^{1} \mathrm{H}$ NMR (DMSO-d $\left.\mathrm{d}_{6}\right): \delta(\mathrm{ppm})=2.50-2.57\left(\mathrm{~m} ; 2 \mathrm{H},-\mathrm{CH}_{2}-\right), 3.61-3.66(\mathrm{~m} ; 2 \mathrm{H}$, $-\mathrm{CH}-), 3.78,3.79$ (2s; $\left.12 \mathrm{H},-\mathrm{OCH}_{3}\right), 6.83-7.07$ (m; 6H, Ar-H), 8.18 (broad $\mathrm{s} ; 6 \mathrm{H},-\mathrm{NH}_{3}{ }^{+}, \mathrm{D}_{2} \mathrm{O}$-exchange), $8.60(\mathrm{~s} ; 4 \mathrm{H}, \mathrm{Ar}-\mathrm{H})$. $-\mathrm{C}_{19} \mathrm{H}_{26} \mathrm{~N}_{2} \mathrm{O}_{4}$. $2 \mathrm{C}_{6} \mathrm{H}_{3} \mathrm{~N}_{3} \mathrm{O}_{7}$ (804.7) Calc. C $46.3 \mathrm{H} 4.01 \mathrm{~N} 13.9$ Found C $46.1 \mathrm{H} 4.15 \mathrm{~N}$ 13.5.

Separation of the 6a-diastereomers by fractional crystallization of their bis-hydrochlorides

After reduction of $4 \mathrm{a}$ and work-up to afford the mixture of $6 \mathrm{a}$-diastereomers, this mixture was freed from EtOAc in vacuo (oil pump), dissolved in absol. EtOH $(300 \mathrm{ml})$ and converted to the bis-hydrochlorides by gaseous $\mathrm{HCl}$ under ice-cooling. After $48 \mathrm{~h}$ at $-25^{\circ} \mathrm{C}$ the crystals were collected, washed with $\mathrm{EtOH}$ and dried in vacuo. meso-6a.2 $\mathrm{HCl}$ so obtained is free from racem.-6a (tlc) and was recrystallized from $\mathrm{AcOH} / \mathrm{H}_{2} \mathrm{O}$ (see above).

The mother liquors were evaporated in vacuo, the residue was dried (oil pump), dissolved in acetone $(200 \mathrm{ml})$, stirred for $30 \mathrm{~min}$ and filtered. After drying racem. $-6 \mathrm{a} \cdot 2 \mathrm{HCl}$, contaminated with meso-6a. $2 \mathrm{HCl}$, was obtained. This mixture was dissolved by boiling in glacial AcOH $(80 \mathrm{ml})$. After $3 \mathrm{~h}$ at room temp. the crystals of contaminating meso- $6 \mathrm{a} \cdot 2 \mathrm{HCl}$ were collected. A further crop of racem. $-6 \mathrm{a} \cdot 2 \mathrm{HCl}$ separated from the filtrate during standing in the refrigerator for $24 \mathrm{~h}$. Partial evaporation and addition of $\mathrm{Et}_{2} \mathrm{O}$ afforded further racem.-6a.2HCl. Recrystallisation from glacial AcOH.

Yields: meso-6a-2HCl: $6.9 \mathrm{~g}(26 \%)$. racem.-6a.2HCl: $6.6 \mathrm{~g} \mathrm{(25 \% ).}$

\section{1,3-Bis-(4'-hydroxyphenyl)-1,3-diaminopropane dihydrobromide (7a)}

meso-7a: meso-6a (1.0 g, 2.8 mmole) was dissolved in $\mathrm{H}_{2} \mathrm{O}(10 \mathrm{ml})$ and dropped into saturated $\mathrm{NaHCO}_{3}$ solution $(30 \mathrm{ml})$. After $30 \mathrm{~min}$ the crystals were filtered, washed with saturated $\mathrm{NaCl}$ solution $(10 \mathrm{ml})$ and $\mathrm{H}_{2} \mathrm{O}(10$ $\mathrm{ml}$ ) and dried in vacuo to afford the free diamine (meso-6a-base). To a stirred solution of meso-6a-base $(0.5 \mathrm{~g}, 1.75 \mathrm{mmole})$ in dry $\mathrm{CHCl}_{3}(50 \mathrm{ml})$ $\mathrm{BBr}_{3}(1.3 \mathrm{ml}, 14.0 \mathrm{mmole})$ in dry $\mathrm{CHCl}_{3}(5 \mathrm{ml})$ was dropped at $-5--10^{\circ} \mathrm{C}$ under $\mathrm{N}_{2}$. The solution was refluxed for $6 \mathrm{~h}$ and stirred at room temp. for $12 \mathrm{~h}$. To the ice-cooled solution dry $\mathrm{CH}_{3} \mathrm{OH}(30 \mathrm{ml})$ was dropped for 30 $\mathrm{min}$, then it was evaporated in vacuo. Acetone $(20 \mathrm{ml})$ was added and the crystals formed were collected. Yield $0.40 \mathrm{~g}(55 \%)$. - m.p. 196-200 $\mathrm{C}$ (dec., after precipitation with $\mathrm{Et}_{2} \mathrm{O}$ from ethanolic solution). - IR $\left(\mathrm{KBr}, \mathrm{cm}^{-1}\right)$ : 2600-3600 (broad, $\mathrm{NH}$ and $\mathrm{OH})$. - ${ }^{1} \mathrm{H}-\mathrm{NMR}\left(\mathrm{D}_{2} \mathrm{O}\right): \delta(\mathrm{ppm})=\mathbf{2 . 5 8 - 2 . 7 6}$ (m; $2 \mathrm{H},-\mathrm{CH}_{2}$ ), 4.26 (dd; $\mathrm{J}_{1}=10.0 \mathrm{~Hz}, \mathrm{~J}_{2}=4.4 \mathrm{~Hz}, 2 \mathrm{H},-\mathrm{CH}-$ ), 6.74 (m; AA'BB', 8H, AR-H). - $\mathrm{C}_{15} \mathrm{H}_{18} \mathrm{~N}_{2} \mathrm{O}_{2} \cdot 2 \mathrm{HBr}(420.2)$ Calc. C $42.9 \mathrm{H} 4.80 \mathrm{~N}$ 6.7 Found $\mathrm{C} 42.9 \mathrm{H} 4.81 \mathrm{~N} \mathrm{6.6}$. - If meso-7a is recrystallized from $\mathrm{AcOH} / \mathrm{H}_{2} \mathrm{O}(4 / 1 ; \mathrm{v} / \mathrm{v})$, the crystals melt at $165-175^{\circ} \mathrm{C}($ dec.) and contain AcOH: $\mathrm{C}_{15} \mathrm{H}_{18} \mathrm{~N}_{2} \mathrm{O}_{2} \cdot 2 \mathrm{HBr} \cdot \mathrm{CH}_{3} \mathrm{COOH}(480.2)$ Calc. C 42.5 H $5.04 \mathrm{~N} 5.8$ Found C 42.4 H 5.29 N 5.7. 
racem.-7a: racem.-6a $(1.0 \mathrm{~g}, 2.8 \mathrm{mmole})$ was dissolved in water $(10 \mathrm{ml})$. After addition of $3 \mathrm{~N}-\mathrm{NaOH}(5 \mathrm{ml})$ racem.-6a-base was extracted with EtOAc ( $3 \times 30 \mathrm{ml}$ \%. After work-up as usual, the free diamine was obtained in $95 \%$ yield as a colourless oil, which crystallizes after drying at room temp. and 0.1 torr. To a stirred solution of racem.-6a-base $(0.5 \mathrm{~g}$, $1.75 \mathrm{mmole})$ in dry $\mathrm{CHCl}_{3}(50 \mathrm{ml}) \mathrm{BBr}_{3}(1.3 \mathrm{ml}, 14.0 \mathrm{mmole})$ in dry $\mathrm{CHCl}_{3}(5 \mathrm{ml})$ was dropped at $-5--10^{\circ} \mathrm{C}$ under $\mathrm{N}_{2}$. The solution was refluxed for $6 \mathrm{~h}$ and stirred at room temp. for $12 \mathrm{~h}$. Dry $\mathrm{CH}_{3} \mathrm{OH}(30 \mathrm{ml})$ was dropped to the ice-cooled solution during $30 \mathrm{~min}$, then it was evaporated completely in vacuo. Acetone $(20 \mathrm{ml})$ was added and the crystals were collected. racem. $-7 \mathrm{a} \cdot 2 \mathrm{HBr}$ was obtained as 0.5 acetone solvate after

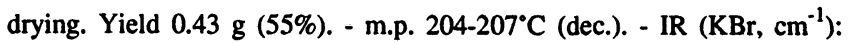
$1700(\mathrm{C}=0) ; 2700-3600$ (broad, $\mathrm{NH}$ and $\mathrm{OH}) .{ }^{1} \mathrm{H}-\mathrm{NMR}\left(\mathrm{D}_{2} \mathrm{O}\right): \delta(\mathrm{ppm})=$ 2.11 (s; $3 \mathrm{H},-\mathrm{CH}_{3}$ ), 2.67 (dd; $\mathrm{J}_{1}=9.3 \mathrm{~Hz}, \mathrm{~J}_{2}=6.9 \mathrm{~Hz}, 2 \mathrm{H},-\mathrm{CH}_{2}$ ), 3.77 (dd; $\left.\mathrm{J}_{1}=9.3 \mathrm{~Hz}, \mathrm{~J}_{2}=6.9 \mathrm{~Hz}, 2 \mathrm{H},-\mathrm{CH}-\right), 6.98\left(\mathrm{~m} ; \mathrm{AA}^{\prime} \mathrm{BB}^{\prime}, 8 \mathrm{H}, \mathrm{Ar}-\mathrm{H}\right)$. $-\mathrm{C}_{15} \mathrm{H}_{18} \mathrm{~N}_{2} \mathrm{O}_{2} \cdot 2 \mathrm{HBr} \cdot 0.5 \mathrm{C}_{3} \mathrm{H}_{6} \mathrm{O}$ (449.2) Calc. C $44.1 \mathrm{H} 5.16 \mathrm{~N}$ 6.2 Found $\mathrm{C}$ 44.1 H 5.35 N 5.9 .

\section{1,3-Bis-(3',4'-dihydroxyphenyl)-1,3-diaminopropane dihydrobromide (7b)}

meso- and racem.-7b were prepared as described for racem.-7a, but the free diamines were extracted with $\mathrm{CH}_{2} \mathrm{Cl}_{2}$ instead of EtOAc.

meso-7b: Yield 53\%. - m.p. $178-183^{\circ} \mathrm{C}$ (dec., $\left.\mathrm{HBr} 62 \% / \mathrm{H}_{2} \mathrm{O}(1 / 1 ; \mathrm{v} / \mathrm{v})\right)$. - IR ( $\left.\mathrm{KBr}, \mathrm{cm}^{-1}\right)$ : $2600-3600$ (broad, NH and OH). - ${ }^{1} \mathrm{H}-\mathrm{NMR}\left(\mathrm{D}_{2} \mathrm{O}\right): \delta$ $(\mathrm{ppm})=2.47-2.80\left(\mathrm{~m} ; 2 \mathrm{H},-\mathrm{CH}_{2}-\right), 4.28\left(\mathrm{dd} ; \mathrm{J}_{1}=10.0 \mathrm{~Hz}, \mathrm{~J}_{2}=4.5 \mathrm{~Hz}, 2 \mathrm{H}\right.$, - $\mathrm{CH}-$ ), 6.48-6.71 (m; 6H, Ar-H). - $\mathrm{C}_{15} \mathrm{H}_{18} \mathrm{~N}_{2} \mathrm{O}_{4} \cdot 2 \mathrm{HBr} \cdot \mathrm{H}_{2} \mathrm{O}$ (470.2) Calc. C 38.3 H 4.27 N 6.0 Found C 38.2 H 4.77 N 5.7.

racem.-7b: Yield 55\%. - m.p. $179-183^{\circ} \mathrm{C}$ (dec., $\mathrm{HBr} 62 \% / \mathrm{H}_{2} \mathrm{O}(1 / 2$; $\mathrm{v} / \mathrm{v}$ )).- IR (KBr, cm $\left.{ }^{-1}\right):$ 2600-3600 (broad, $\mathrm{NH}$ and OH). - ${ }^{1} \mathrm{H}-\mathrm{NMR}\left(\mathrm{D}_{2} \mathrm{O}\right)$ : $\delta(\mathrm{ppm})=2.64\left(\mathrm{dd} ; \mathrm{J}_{1}=9.0 \mathrm{~Hz}, \mathrm{~J}_{2}=3.0 \mathrm{~Hz}, 2 \mathrm{H},-\mathrm{CH}_{2}-\right), 3.75\left(\mathrm{dd} ; \mathrm{J}_{1}=9.0\right.$ $\left.\mathrm{Hz}, \mathrm{J}_{2}=3.0 \mathrm{~Hz}, 2 \mathrm{H},-\mathrm{CH}-\right), 6.59-6.96(\mathrm{~m} ; 6 \mathrm{H}, \mathrm{Ar}-\mathrm{H}) .-\mathrm{C}_{15} \mathrm{H}_{18} \mathrm{~N}_{2} \mathrm{O}_{4} \cdot 2 \mathrm{HBr}$ (452.1) Calc. C 39.9 H 4.46 N 6.2 Found C 39.8 H 4.40 N 6.1.

\section{Diiodo-1,3-bis-(4'-hydroxyphenyl)-1,3-diaminopropane-platinum(II) (2a)}

meso-2a: $\mathrm{K}_{2} \mathrm{Ptl}_{4}$ solution was prepared by stirring $\mathrm{K}_{2} \mathrm{PtCl}_{4}(0.104 \mathrm{~g}, 0.25$ mmole) and $\mathrm{KI}(0.375 \mathrm{~g})$ in $\mathrm{H}_{2} \mathrm{O}(2.5 \mathrm{ml})$ for $30 \mathrm{~min}$ at room temp. meso-7a.AcOH $(0.12 \mathrm{~g}, 0.25 \mathrm{mmole})$ was dissolved in $12 \mathrm{ml}$ of $\mathrm{H}_{2} \mathrm{O} /$ tert. butanol $(1 / 1 ; \mathrm{v} / \mathrm{v})$, and the $\mathrm{pH}$-value was adjusted to $6.0-6.5$ by $0.1 \mathrm{~N}$ $\mathrm{NaOH}$. $\mathrm{K}_{2} \mathrm{PtI}_{4}$ solution was dropped to this solution during $30 \mathrm{~min}$ at $40^{\circ} \mathrm{C}$. The mixture was stirred at $40^{\circ} \mathrm{C}$ and $\mathrm{pH}$ 6.0-6.5 for $4 \mathrm{~h}$. After further $3 \mathrm{~h}$ at room temp. the yellow coloured product was filtered, washed with water and dried in vacuo. Yield $0.171 \mathrm{~g}(97 \%)$ - - m.p. 199-201 ${ }^{\circ} \mathrm{C}$ (dec.). - IR $\left(\mathrm{KBr}, \mathrm{cm}^{-1}\right):$ 2800-3600 (broad, NH and OH). - ${ }^{1} \mathrm{H}-\mathrm{NMR}$ (DMF-d $): \delta$ $(\mathrm{ppm})=2.02-2.18\left(\mathrm{~m} ; 1 \mathrm{H},-\mathrm{CH}_{2}-\right), 2.46-2.75\left(\mathrm{~m} ; 1 \mathrm{H},-\mathrm{CH}_{2}-\right), 4.32-4.52(\mathrm{~m}$; $2 \mathrm{H},-\mathrm{CH}-), 4.60-4.85\left(\mathrm{~m} ; 2 \mathrm{H},-\mathrm{NH}_{2}\right), 5.30-5.70\left(\mathrm{~m} ; 2 \mathrm{H},-\mathrm{NH}_{2}\right), 7.12(\mathrm{~m}$; AA'BB', 8H, Ar-H), 9.70 (s; 2H, Ar-OH). - PI-FAB-MS (glycerol/DMSO; $\left.{ }^{194} \mathrm{Pt}\right): \mathrm{m} / \mathrm{z}=657\left(85 \%,(\mathrm{M}-\mathrm{I}+\mathrm{DMSO})^{+}\right) .-\mathrm{C}_{15} \mathrm{H}_{18} \mathrm{I}_{2} \mathrm{~N}_{2} \mathrm{O}_{2} \mathrm{Pt}(707.2)$ Calc. C 25.5 H 2.57 N 4.0 Found C 25.2 H 2.68 N 3.7.

racem.-2a: same procedure as in the synthesis of meso-2a with racem.-7a (0.104 g, 0.25 mmole). Yield $0.163 \mathrm{~g}(92 \%)$. - m.p. 206-208 ${ }^{\circ} \mathrm{C}$. - IR (KBr, $\left.\mathrm{cm}^{-1}\right): 2800-3600$ (broad, NH and OH). - ${ }^{1} \mathrm{H}-\mathrm{NMR}\left(\mathrm{DMF}-\mathrm{d}_{7}\right): \delta(\mathrm{ppm})=$ 2.42-2.52 (m; $\left.2 \mathrm{H},-\mathrm{CH}_{2}-\right)$, 4.10-4.27 (m; $\left.2 \mathrm{H},-\mathrm{CH}-\right), 5.14-5.30(\mathrm{~m} ; 4 \mathrm{H}$, - $\mathrm{NH}_{2}$ ), 7.24 (m, AA'BB', 8H, Ar-H), 9.69 (s; 2H, Ar-OH). - PI-FAB-MS (glycerol/DMSO; $\left.{ }^{194} \mathrm{Pt}\right): \mathrm{m} / \mathrm{z}=657\left(85 \%,(\mathrm{M}-\mathrm{I}+\mathrm{DMSO})^{+}\right)$. $\mathrm{C}_{15} \mathrm{H}_{18} \mathrm{I}_{2} \mathrm{~N}_{2} \mathrm{O}_{2} \mathrm{Pt}(707.2)$ Calc. C $25.5 \mathrm{H} 2.57 \mathrm{~N} 4.0$ Found C $25.2 \mathrm{H} 2.57 \mathrm{~N}$ 3.8 .

Diiodo-1,3-bis-(3',4'-dihydroxyphenyl)-1,3-diaminopropane-platinum(II) (2b)

meso-2b and racem.-2b were prepared as described for meso-2a. meso-2b: Yield $0.073 \mathrm{~g} \mathrm{(39 \% ).} \mathrm{-} \mathrm{m.p.} 166-169^{\circ} \mathrm{C}$ (dec.). - IR (KBr, $\mathrm{cm}^{-1}$ ): 2900-3600 (broad, NH and OH). - ${ }^{1}$ H-NMR (DMF-d $): \delta(\mathrm{ppm})=$ 2.02-2.15 (m; $\left.1 \mathrm{H},-\mathrm{CH}_{2}-\right), 2.48-2.66\left(\mathrm{~m} ; 1 \mathrm{H},-\mathrm{CH}_{2}-\right), 4.24-4.46(\mathrm{~m} ; 2 \mathrm{H}$, -CH-), 4.50-4.66 (m; 2H, $\left.-\mathrm{NH}_{2}\right), 5.32-5.66\left(\mathrm{~m} ; 2 \mathrm{H},-\mathrm{NH}_{2}\right), 6.78-7.10(\mathrm{~m}$; $6 \mathrm{H}, \mathrm{Ar}-\mathrm{H}$ ), 9.12 (s; $4 \mathrm{H}, \mathrm{Ar}-\mathrm{OH}) .-\mathrm{C}_{15} \mathrm{H}_{18} \mathrm{I}_{2} \mathrm{~N}_{2} \mathrm{O}_{4} \mathrm{Pt}$ (739.2) Calc. C $24.4 \mathrm{H}$ $2.45 \mathrm{~N} 3.8$ Found $\mathrm{C} 24.2 \mathrm{H} 3.14 \mathrm{~N} 3.6$.

racem.-2b: Yield $0.172 \mathrm{~g}(93 \%)$ - m.p. $170-172^{\circ} \mathrm{C}$ (dec.). - IR (KBr, $\left.\mathrm{cm}^{-1}\right): 2900-3600$ (broad, NH and OH). $-{ }^{1} \mathrm{H}-\mathrm{NMR}$ (DMF-d $): \delta(\mathrm{ppm})=$ 2.34-2.48 (m; $\left.2 \mathrm{H},-\mathrm{CH}_{2}-\right)$, 3.96-4.34 (m; $\left.2 \mathrm{H},-\mathrm{CH}-\right), 4.90-5.40(\mathrm{~m} ; 4 \mathrm{H}$, $-\mathrm{NH}_{2}$ ), 6.78-7.22 (m; 6H, Ar-H), $9.05(\mathrm{~s} ; 4 \mathrm{H}, \mathrm{Ar}-\mathrm{OH}) .-\mathrm{C}_{15} \mathrm{H}_{18} \mathrm{I}_{2} \mathrm{~N}_{2} \mathrm{O}_{4} \mathrm{Pt}$ (739.2) Calc. C 24.4 H 2.45 N 3.8 Found C 24.4 H 2.69 N 3.8.

\section{References}

1 H. Schönenberger, R. Gust, J. Karl, Th. SpruB, M.R. Schneider, R.W. Hartmann, Ch. Batzel, S. Schertl, F. Lux, and S. Trebert-Haeberlin, "Rezeptorgebundene Chemotherapie" in "Antiöstrogene in Forschung und Klinik“, p. 71, Reihe: "Aktuelle Onkologie“ Zuckschwerdt Verlag, München 1989, and lit. cited therein.

2 M. Jennerwein, R. Gust, R. Müller, H. Schönenberger, J. Engel, M.R. Berger, D. Schmähl, S. Seeber, R. Osieka, G. Atassi, and D. MaréchalDe Bock, Arch. Pharm. (Weinheim) 322, 67 (1989).

3 Idem, Arch. Pharm. (Weinheim) 322, 25 (1989).

4 E.P. Kohler and H.M. Chadwell, Org. Synthesis, Coll. Vol. 1, 2. ed., p. 78, John Wiley and Sons, Inc., New York ... 1967.

5 S. Arakawa, K. Kashiwabara, J. Fujita, and K. Saito, Bull. Chem. Soc. Jpn. 50, 2108 (1977). - Probably the legends of Fig. 1 on page 2109 have been exchanged inadvertantly: according to F.A. Bovey, Nuclear Magnetic Resonance Spectroscopy, 2nd ed., p. 130, Academic Press, Inc., San Diego .... 1988, "the fluorine nuclei of the central $\mathrm{CF}_{2}$ group in meso- $\mathrm{CF}_{2} \mathrm{Cl}-\mathrm{CFCl}-\mathrm{CF}_{2}-\mathrm{CFCl}-\mathrm{CF}_{2} \mathrm{Cl}$ have differing chemical shifts and couplings, whereas those of the racemic isomer are identical." This is corroborated by symmetry considerations for meso- and racemic 2,4-diaminoglutaric acid (M. Hesse, H. Meier, B. Zeeh, Spektroskopische Methoden in der organischen Chemie, 3rd ed., p. 85, Thieme Verlag, Stuttgart 1987): in the meso-form the $\mathrm{CH}_{2}$ protons are diastereotopic, whilst they are homotopic in the racemate. - In our compounds 6 the differences of the ${ }^{1} \mathrm{H}-\mathrm{NMR}$-spectra are less pronounced than in the bis-amides 5 .

6 K.v. Auwers and H. Müller, J. Prakt. Chem. N.F. 137, 57 (1933).

7 S. Kano, Y. Tanaka, E. Sugino, and S. Hibino, Synthesis 1980, 695.

8 F. Straus, Liebigs Ann. Chem. 374, 121 (1910).

9 T. Richardson, R. Robinson, and E. Seijo, J. Chem. Soc. 1937, 837.

[Ph771] 